\title{
The Role of Phakic Intraocular Lens Implants in Treatment of High-Refractive Errors and Amblyopia in Children
}

Sarah Moran • Michael O'Keefe

To view enhanced content go to www.ophthalmology-open.com

Received: January 30, 2013 / Published online: March 26, 2013

(C) The Author(s) 2013. This article is published with open access at Springerlink.com

\section{ABSTRACT}

Introduction: This article aims to evaluate the role of phakic intraocular lens (pIOL) implants in the treatment of high-refractive errors and amblyopia in the pediatric setting.

Methods: A literature search of the PubMed database was conducted in December 2012 using the keywords: pIOL implants, amblyopia, pediatric refractive surgery and ametropia, and articles of high or medium clinical relevance were selected for review. Inclusion criteria were children aged $0-17$ years who had undergone pIOL implantation for the treatment of refractive errors and/or amblyopia.

Results: Multiple studies have shown the relevancy and effectiveness of pIOL implantation as an alternative surgical management for highly

S. Moran $(\bowtie) \cdot$ M. O'Keefe

Department of Ophthalmology,

The Children's University Hospital,

Temple Street, Dublin 2, Ireland

e-mail: sarmoran@gmail.com

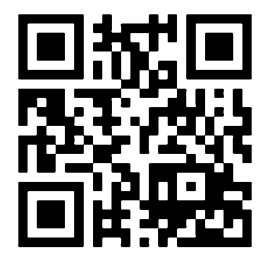

Enhanced content for this article is

available on the journal web site:

www.ophthalmology-open.com significant pediatric ametropia in selective patients who are noncompliant with medical treatment.

Conclusions: In the management of clinically significant severe pediatric ametropic and/or anisometropic myopia or hyperopia and in the event of nonadherence to traditional medical treatment, phakic anterior chamber IOL implantation is currently considered an effective modality of treatment. Long-term follow-up of pediatric patients following pIOL implantation is necessary. Future clinical trials are needed to address the safety and efficacy of the type of and the best time for pIOL implantation in treatment and/or prevention of amblyopia in children.

Keywords: Amblyopia; Ametropia;

Pediatric refractive surgery;

Phakic intraocular lens implants

\section{INTRODUCTION}

Refractive surgery in children is controversial. Most children with refractive errors can be treated adequately and safely with spectacles or contact lenses, along with conventional 
therapy for amblyopia. However, in specific subpopulations of children, intervention may be necessary to achieve full visual function and prevent amblyopia. Procedures employed in pediatric refractive surgery include laser keratorefractive procedures and intraocular procedures such as phakic intraocular lens (pIOL) implantation or clear lens extraction. There is an abundance of literature demonstrating the efficacy and safety of pIOLs in the adult population $[1,2]$. In children, however, there is less evidence to support their use. This article aims to review the evidence for pIOLs in the treatment of high-refractive errors and amblyopia in the pediatric population.

\section{Indication for pIOLs in Children}

Most children with refractive errors can be treated adequately and safely with spectacles or contact lenses, along with conventional therapy for amblyopia. However, in certain subsets of children, traditional treatment is ineffective and further intervention is necessary. Currently, refractive surgery in children is performed only when conventional treatments have failed. In children with high-refractive errors, spectacle tolerance can be reduced secondary to prismatic induced aberrations, limited field of view, as well as cosmetic appearance. Contact lenses can also be problematic in children due to difficulty with insertion and removal, infection, intolerance and expense.

The particular pediatric subpopulations that may require refractive surgery include those with bilateral significant ametropia or anisometropia who are spectacle or contact lens noncompliant, as well as those with secondary high-refractive amblyopia in children with neurobehavioral disorders (Table 1).
Table 1 Indications for phakic intraocular lens implantation in children

1. High anisometropia, myopia, or hyperopia noncompliant with conventional treatment

2. Bilateral high ametropia noncompliant with conventional treatment

3. Secondary high-refractive amblyopia associated with neurobehavioral disorders

\section{pIOL Implants}

pIOLs are available in three models, iris-fixated anterior chamber, ciliary sulcus-supported posterior chamber, or angle-supported anterior chamber. The preference currently appears to be for iris-fixated anterior chamber IOLs [3]. The first pIOL implanted in a child was carried out in 1998 by Lesueur and Arne [4], a posterior chamber pIOL (PC-pIOL) was successfully implanted in five children (aged 3-16 years) with high anisometropic myopia who were noncompliant with spectacles or contact lenses. Following the positive outcome of this study, further studies were undertaken using both anterior and PC-pIOLs to restore emmetropia [3-5, 9-15], with the aim of treating refractive-induced amblyopia.

\section{PC-pIOL}

Lesueur and Arne [4] reported results of PC-pIOL insertion in five eyes of children aged 3-16 years old with amblyopic high myopia and a mean preoperative spherical equivalent of $-12.8 \mathrm{D}$. There were no complications reported from the study, and all parents reported an improvement in their child's quality of life. A gain of 3 or more Snellen lines, as well as recovery of binocular vision was observed in two patients. A follow-up study by Lesueur and 
Table 2 Pediatric anterior chamber phakic intraocular lens studies

\begin{tabular}{lclcc}
\hline Author & Eyes & Indication & Age & Follow-up \\
\hline Chipont et al. [11] & 1 & Unilateral high myopia & 8 & 18 months \\
Saxena et al. [12] & 1 & Unilateral high myopia & 4 & 3 years \\
Lifshitz and Levy [13] & 1 & Unilateral high myopia (PC-pIOL) & 3 & 9 months \\
Assil et al. [15] & 1 & Unilateral high myopia & 3 & 4 years \\
Tychsen et al. [16] & 20 & Bilateral high ametropia (16 eyes) and high & $4-17$ & $3-15$ months \\
& & anisometropia (4 eyes) in neurobehavioral disorders & $5-11$ & 3 years \\
Pirouzian and Ip [14] & 7 & Unilateral high myopia & $2-15$ & 5 years \\
Alio et al. [7] & 10 & High anisometropia and amblyopia & $8-15$ & 15 months \\
Ryan et al. [17] & 11 & High bilateral myopia in neurobehavioral & disorders, high anisometropia & $2-17$ \\
\end{tabular}

Data from $[7,11-17]$

$P C$-pIOL posterior chamber phakic intraocular lens

Arne [5] reported outcomes of PC-pIOL to correct high myopia and amblyopia in 12 eyes of children aged 3-16 years old. These children had a mean preoperative spherical equivalent of $-12.7 \mathrm{D}$ and were followed up for a period of 20.5 months. Six patients recovered binocular vision and showed improvement in QOL. There were no reported complications.

BenEzra et al. [6] implanted PC-pIOLs in three children (aged 9-18 years) with anisometropic amblyopia and myopia of -6 to -16 D. Follow-up over a 9-month period showed significant improvement in visual acuity and binocular function. No change in endothelial cell count was observed over the 9-month follow-up period.

Alio et al. report the longest follow-up period of 5 years, following PC-pIOL implantation in a child with high anisometropic amblyopia. Improvement in visual acuity of one logMAR line was reported, and no complications were seen [7].

Potential complications of PC-pIOLs include cataract formation, pupillary block glaucoma, posterior dislocation into the vitreous cavity, and retinal detachment [8]. Reports of complications in the pediatric population are rare; however, this is likely due to the low overall numbers of PC-pIOLs in children.

\section{Anterior Chamber Iris-Claw pIOL}

The iris-claw anterior chamber IOL, designed by Jan Worst, was first implanted in the adult population in 1978 to correct aphakia following cataract surgery. In 1986, this design was altered to a biconcave polymethyl-methacrylate optic with an overall length of $8.5 \mathrm{~mm}$, and the IOL was implanted in the first sighted myopic eye [9]. The design was further modified in 1991 to a convex-concave IOL, and in 1998, the name of the lens was changed to Artisan ${ }^{\circledR}$ (Ophtec B.V., Groningen, The Netherlands) and Verisyse $^{\mathrm{TM}}$ (Abbott Medical Optics, Abbott Laboratories Inc. Abbott Park, IL, USA). The iris-claw IOL was first used in children in 1997 to correct aphakia in a series of 27 children (38 eyes) [10]. 
Studies on pediatric anterior chamber pIOL are shown in Table 2 [7, 11-17]. The use of iris-claw anterior chamber IOLs expanded to treatment of anisometropic amblyopia in children when Chipont et al. [11] implanted an Artisan pIOL in an 8-year-old child, achieving a corrected visual acuity of 20/25 which was stable at 18-month follow-up. No complications were reported. Saxena et al. [12] showed an improvement of visual acuity to 20/20 in a 4-year-old child with Artisan pIOL implantation for high myopic anisometropia. A significant rate of $11.9 \%$ endothelial cell loss was observed. Lifshitz and Levy [13] observed positive results of improved visual acuity following implantation of Artisan pIOL in a 14-month-old pseudophakic child over a 9-month follow-up period. Pirouzian and Ip [14] implanted iris-fixated pIOLs in seven children with anisometropic myopia and amblyopia. Three-year results showed improvement in visual acuity of more than six lines in all patients and no postoperative complications were reported. Assil et al. [15] reported positive 4-year follow-up results in 3 years old with myopic anisometropia. Tychsen et al. [16] reported a larger series of pIOLs in 12 children with neurobehavioral disorders and high ametropia, and found that visual function was substantially improved by an average of $73 \%$ in bilateral ametropic patients and $58 \%$ in anisometropic patients. Ryan et al. [17] observed a significant improvement in vision and vision-related QOL in 11 eyes of six children implanted with Artiflex $^{\circledR}$ (Ophtec B.V., Groningen, The Netherlands) pIOL for high bilateral myopia and concurrent neurobehavioral disorders, high anisometropia, or high myopic astigmatism. There were no intraoperative or postoperative complications observed. Five-year follow-up of nine children implanted with iris-fixated pIOL for anisometropic amblyopia was reported by Alio et al. [7]. Improvement of more than three logMAR lines of corrected visual acuity was seen in all nine children. Endothelial cell count at 5 years postoperatively was $>2,000$ cells $/ \mathrm{mm}^{2}$ in $80 \%$ of patients; for the remaining patients, eye rubbing and ocular trauma was a factor in endothelial cell loss.

\section{DISCUSSION}

Management of pediatric high bilateral ametropia and anisometropia is challenging. In cases where spectacle or contact lens correction is not tolerated, the child is at risk of secondary high-refractive amblyopia. Management options in such cases include corneal laser ablative procedures, refractive lens exchange, and pIOL insertion.

The advantages of pIOLs are numerous (Table 3). With pIOLs, the surgeon is capable of treating a much larger range of refractive errors than can be safely treated with corneal refractive surgery, and natural accommodation is preserved. The skills required for insertion are

Table 3 Advantages of phakic intraocular lenses

1. Reversibility and ability to exchange IOL

2. Predictability

3. High visual quality

4. Quick visual rehabilitation

5. Preservation of accommodation

6. Preservation of corneal surface-option of bioptics

7. Lack of regression

8. No special operating room requirements

9. Can be performed by nonrefractive surgeons

10. Retinal magnification in myopic eyes-potential gain of vision

IOL intraocular lens 


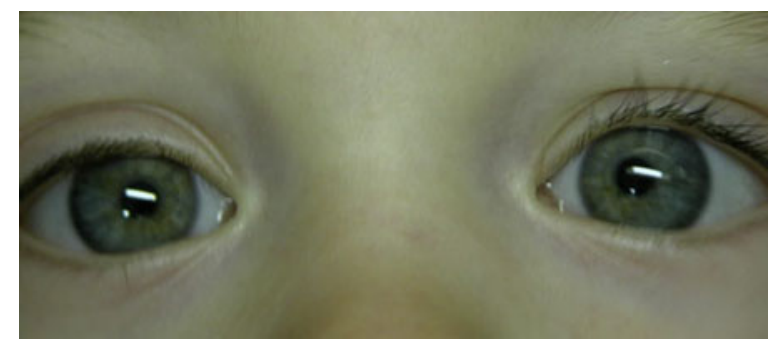

Fig. 1 Child with bilateral iris-fixated anterior chamber phakic intraocular lens

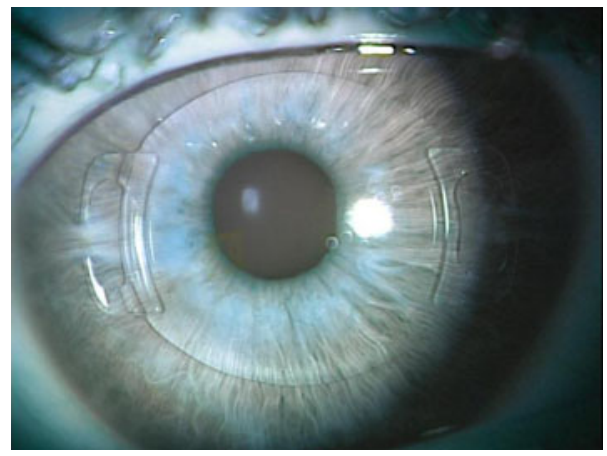

Fig. 2 Artiflex iris-fixated phakic intraocular lens in situ

similar to those required for cataract surgery, and the equipment is significantly less expensive. In comparison, corneal laser ablation carries the disadvantage of corneal haze and high rate of refractive regression with photorefractive keratectomy; particularly as large refractive errors are being treated [18, 19]. LASIK is technically difficult in children, requires general anesthesia, and has the risk of long-term ectasia. Traumatically induced displacement of a LASIK flap is a serious and sight-threatening complication, which children are inherently at high risk of given the nature of children's behavior and activities. Refractive lens exchange results in an increased risk of retinal detachment, glaucoma, and posterior capsule opacification, as well as loss of accommodation [20].

The implant of choice for pediatric patients is the anterior chamber Artisan iris-fixated pIOL

(Figs. 1, 2), although the foldable Artiflex model which requires a smaller incision and less corneal sutures has been shown to be safe and effective also [17]. Safe insertion and lower long-term risk for endothelial cell loss requires an anterior chamber depth of $3.2 \mathrm{~mm}$ or greater. This may preclude the use of irisfixated pIOLs in children younger than 3 years, and children with shallow anterior chambers, as can occur in children with high myopia following retinopathy of prematurity. An iridotomy or iridectomy must be performed during the procedure to reduce chances of pupillary block. In cases of high bilateral ametropia, the eyes are usually implanted sequentially, about 1 month apart. Absorbable sutures are used to avoid re-anesthetizing the child for suture removal.

On review of the published literature, the results show that pIOLs are an effective option in pediatric patients to improve visual acuity and prevent amblyopia. The visual outcome in these patients cannot be compared with the standard IOL patient due to the underlying pathologies. Lack of $20 / 20$ vision is usually a result of numerous factors such as intractable amblyopia, optic nerve disorders, retinopathies, or foveopathies.

Reports of adverse events in the pediatric population are rare due to the limited number of surgeries performed; however, complications in adults include cataract formation, uveitis, IOL dislocation, pigment dispersion, and

Table 4 Complications of phakic intraocular lenses

1.

2.

3.

4.

5.
Endothelial cell loss

Dislocation

Pigment dispersion

Cataract

Shallow anterior chamber 
endothelial cell loss (Table 4). The most significant concern in the pediatric population is long-term corneal endothelial cell loss. This is particularly important given the long life expectancy of the child, as well as potential for eye rubbing. However, experience in the pediatric population to date is limited and this is compounded by difficulties in obtaining accurate endothelial cell counts in children. In the studies of Pirouzian and Ip [14] and Alio et al. [7], the endothelial cell loss rate varied over the course of $3-5$ years between $6.5 \%$ and $15.2 \%$.

\section{CONCLUSION}

Early intervention is necessary in a small subset of the pediatric population with high anisometropia, high bilateral ametropia, or secondary high-refractive amblyopia who are noncompliant with traditional therapy. Careful patient selection and follow-up are essential and further studies are necessary to determine the long-term safety in the pediatric population. pIOLs have been shown to improve visual acuity and vision-related quality of life in children, and plays an increasingly important role in the treatment of high-refractive errors and amblyopia.

\section{ACKNOWLEDGMENTS}

Dr. Sarah Moran is the guarantor for this article, and takes responsibility for the integrity of the work as a whole. No sponsorship was received and there are no financial interests to declare.

Conflict of interest. Sarah Moran and Michael O'Keefe declare no conflict of interest.
Open Access. This article is distributed under the terms of the Creative Commons Attribution Noncommercial License which permits any noncommercial use, distribution, and reproduction in any medium, provided the original author(s) and the source are credited.

\section{REFERENCES}

1. Huang DH, Schalhorn SC, Sugar A, Farjo AA, Majmudar PA, Trattler WB, Tanzer DJ. Phakic intraocular lens implantation for the correction of myopia. A report by the American Academy of Ophthalmology. Ophthalmology. 2009;116:2244-58.

2. Budo C, Hessloehl JC, Izak M, Luyten GPM, Menezo JL, Sener BA, Tassignon MJ, Termote H, Worst JGF. Multicenter study of the Artisan phakic intraocular lens. J Cataract Refract Surg. 2000;26:1163-71.

3. Pirouzian A. Pediatric phakic intraocular lens surgery: review of clinical studies. Curr Opin Ophthalmol. 2010;21:249-54.

4. Lesueur LC, Arne JL. Phakic posterior chamber lens intraocular implantation in children with high myopia. J Cataract Refract Surg. 1999;25:1571-5.

5. Lesueur LC, Arne JL. Phakic intraocular lens to correct high myopia amblyopia in children. J Refract Surg. 2002;18:519-23.

6. BenEzra D, Cohen E, Karshai I. Phakic posterior chamber intraocular lens for the correction of anisometropia and correction of amblyopia. Am J Ophthalmol. 2000;130:292-6.

7. Alio JL, Toffaha BT, Laria C, Pinero DP. Phakic intraocular lens implantation for treatment of anisometropia and amblyopia in children: 5-year follow-up. J Refract Surg. 2011;27:494-501.

8. Eleftheriadis H. Potential complications of phakic IOLs. Br J Ophthalmol. 2004;88:1480-1.

9. Fechner PU, Worst JGF. A new concave intraocular lens for the correction of myopia. Eur J Implant Refract Surg. 1989;1:41-3.

10. van der Pol BA, Worst JG. Iris-Claw intraocular lenses in children. Doc Ophthalmol. 1996-1997; 92:29-35.

11. Chipont EM, García-Hermosa P, Alió JL. Reversal of myopic anisometropic amblyopia with phakic 
intraocular lens implantation. J Refract Surg. 2001; $17: 460-2$.

12. Saxena R, van Minderhout HM, Luyten GP. Anterior chamber iris-fixated phakic intraocular lens for anisometropic amblyopia. J Cataract Refract Surg. 2003;29:835-8.

13. Lifshitz T, Levy J. Secondary artisan phakic intraocular lens for correction of progressive high myopia in a pseudophakic child. J AAPOS. 2005;16:151-7.

14. Pirouzian A, Ip KC. Anterior chamber phakic intraocular lens implantation in children to treat severe anisometropic myopia and amblyopia: 3-year clinical results. J Cataract Refract Surg. 2010;9:1486-93.

15. Assil KK, Sturm JH, Chang SH. Verisyse intraocular lens implantation in a child with anisometropic amblyopia: four-year follow-up. J Cataract Refract Surg. 2007;33:1985-6.
16. Tychsen L, Hoekel J, Ghasia F, Yoon-Huang G. Phakic intraocular lens correction of high ametropia in children with neurobehavioral disorders. J AAPOS. 2008;12:282-9.

17. Ryan A, Hartnett C, Lanigan B, O'Keefe M. Foldable iris-fixated intraocular lens implantation in children. Acta Ophthalmol. 2012;90:458-62.

18. O'Keefe M, Nolan L. LASIK surgery in children. Br J Ophthalmol. 2004;88:19-21.

19. Paysse EA. Photorefractive keratectomy for anisometropic amblyopia in children. Trans Am Ophthalmol Soc. 2004;102:341-72.

20. Horgan N, Condon P, Beatty S. Refractive lens exchange in high myopia: long-term follow-up. Br J Ophthalmol. 2005;89:670-2. 